\title{
Novel design of a one-dimensional measurement probe
}

Rudolf Thalmann, Dannis M. Brouwer, Han Haitjema, Piet H. J. Schellekens

Rudolf Thalmann, Dannis M. Brouwer, Han Haitjema, Piet H. J. Schellekens, "Novel design of a one-dimensional measurement probe," Proc. SPIE 4401, Recent Developments in Traceable Dimensional Measurements, (22 October 2001); doi: 10.1117/12.445617

SPIE. Event: Lasers in Metrology and Art Conservation, 2001, Munich, Germany 


\title{
Novel design of a one-dimensional measurement probe
}

\author{
R. Thalmann*, D.M. Brouwer**, H. Haitjema**, P.H.J. Schellekens** \\ * Swiss Federal Office of Metrology and Accreditation; ** Technical University Eindhoven
}

\begin{abstract}
The mechanical probing system is often one of the limiting factors in the calibration of length standards. It has been shown, that for highly accurate applications a particular effect, which is often not considered, has to be taken into account: a spherical probe on a stylus undergoes a small rotation due to the angular stylus deflection, which creates friction and potentially stick slip during the probing process and may thus lead to non-reproducible probing. A novel probe has been built which avoids this effect by an additional degree of freedom, providing a small vertical movement of the stylus. The probe is of a monolithic flexure hinge design with a rigid connection of the stylus and the mirror reflector for the plane mirror interferometer, which measures the displacement. The measurement force, which is proportional to the deflection of the hinges, is measured with a capacitive probe. The probing procedure generates the force/deflection curve and allows for the measurement force to be extrapolated to zero. The presented test results show the system's capability for a probing accuracy in the nanometer range.
\end{abstract}

Keywords: Mechanical probe, length measurement

\section{INTRODUCTION}

For the calibration of length artefacts, the probing system is a key element. Most often, probing is done by mechanical contact of the stylus with the measurement surface. Two usually independent measurement systems have to be appropriately combined: a (primary) scale, measuring the displacement of the entire probing system and a (secondary) transducer, measuring the probe deflection and thus the probing force. Ideally, the overall system should not be limited by the transducer, but by the primary length measurement device. Dependent on the measurement task, the probe may have one or several degrees of freedom. Whereas for co-ordinate measuring machines three-dimensional probes are used, for length measuring machines a one-dimensional probe is more appropriate.

\section{PRINCIPAL DESIGN REQUIREMENTS}

The purpose of the development described hereafter was the realization of a highly accurate probe for a one-dimensional length measurement machine. The essential requirements were:

- The probe had to fit on an existing length measurement machine [1] and shall be applied for the calibration of internal and external diameter standards.

- The probe shall provide high resolution, repeatability and stability, all within a few nanometers.

- The resulting probing point must be free of any influence due to measurement forces or other contacting effects.

In addition, good metrology practice and the design of the existing machine imposed the following requirements:

- The Abbe principle must be fulfilled.

- For the measurement, the probe is the moving part, and not the artefact to be measured.

- The probe displacement is measured by a plane mirror interferometer.

- Spherical probing elements shall be used.

These requirements determined the principle design as it is sketched in Figure 1. The two metrological circles are to be in line (Abbe principle) and as compact and stable as possible. The fixed circle is formed by the artefact to be calibrated, which is firmly connected to the interferometer. The laser beam of the interferometer and the measured diameter are coaxial.

* Swiss Federal Office of Metrology and Accreditation (METAS), Lindenweg 50, CH-3003 Bern-Wabern, Switzerland, rudolf.thalmann@metas.ch,www.metas.ch

** Technical University Eindhoven, P.O. Box 513, NL-5600 MB Eindhoven, Netherlands. 
The stylus together with the plane mirror reflector form the second, moving circle. The possibility to vertically displace the stylus/mirror assembly and thus to move around the artefact allows also the measurement of external diameter standards.

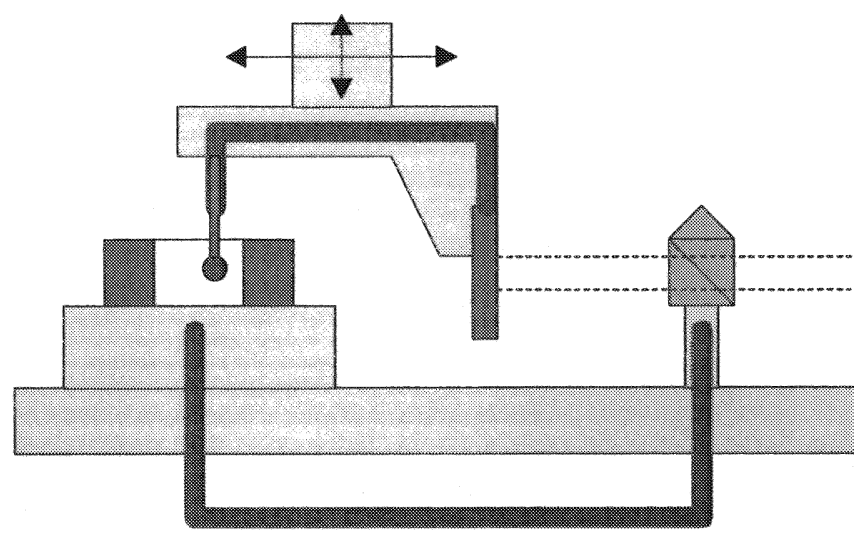

Figure 1: Principle of a probing system fulfilling the Abbe principle with compact metrological circles.
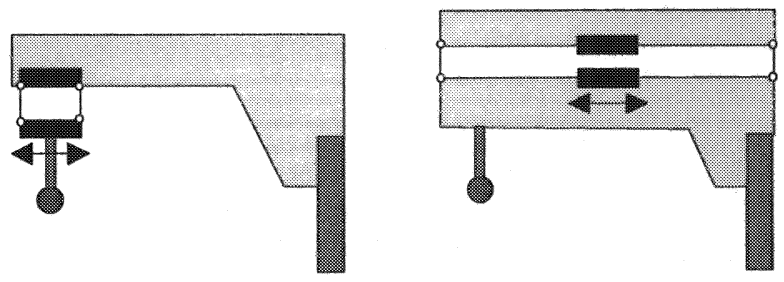

Figure 2: Two probing system principles with deflecting (left) and rigid (right) stylus to mirror link.

The detection of the mechanical contact is always a measurement of the force exerted on the probing element. This can be accomplished in two ways: The usual method is by measuring the displacement of a moveable stylus with respect to the frame holding the mirror [1,2], e.g. using an inductive probe (Figure 2, left). This sets very high requirements to the probe. An alternative way is to have the stylus rigidly connected to the mirror [3] and allowing for an elastic movement of this assembly with respect to the ram (Figure 2, right). The contact force is then measured by the displacement of the assembly. The latter design has the advantage, that due to the stiffness of the stylus/mirror connection, the deflection of the stylus due to the measurement force will be much smaller than the deflection of the hinges and consequently the accuracy requirements for the displacement sensor are much reduced compared to the former design. Assuming the force be measured by a displacement, the gain is in fact given by the ratio of the displacement to be measured and the stylus bending, or by the ratio of the stiffness of the stylus/mirror assembly to the stiffness of the elastic hinge holding this frame. This stiffness can in principle be almost arbitrarily chosen and will be determined by optimizing the weight and the dynamic behavior of the system. The above mentioned ratio for the probe described hereafter is about 20.

\section{FRICTION AT STYLUS CONTACT}

During stylus deflection, the contact point of the probe sphere would normally experience a small vertical movement which causes friction forces. Three mechanisms are responsible for this vertical movement:

- The stylus bending causes the probe sphere to describe a nearly circular path.

- Bending of the elastic hinges makes the stylus/mirror frame to describe a nearly circular path.

- Bending of the stylus yields a rotation of the sphere which causes the sphere to roll vertically along the object in upward direction.

It can be shown, that the latter effect is the most significant. From the theory of elastic bending of a cylindrical shaft, the rotation angle $\phi$ of the sphere is given by

$$
\phi \approx \tan \phi=\frac{3 x_{b}}{2 l}=\frac{3 F}{2 l c},
$$

where $x_{b}$ denotes the stylus bending caused by the measurement force $F$ and $c$ is the stiffness of the stylus (Figure 3). Assuming a stylus with a cylindrical shaft of length $l$ and diameter $d$, the stiffness is given by 


$$
c=\frac{3 \pi d^{4} E}{64 l^{3}},
$$

with $E$ being the module of elasticity of the stylus shaft. A numerical example with a tungsten carbide stylus of length $l=20 \mathrm{~mm}$ and diameter $d=2 \mathrm{~mm}$ shows, that the contact point on a probe sphere of radius $r=2 \mathrm{~mm}$ will try to rotate by $z=r \phi=0.5 \mu \mathrm{m}$ for a measurement force of $0.5 \mathrm{~N}$.

The friction force at the rotating contact point produces a friction moment $M_{W}=r \cdot \mu \cdot F$, where $\mu$ is the friction coefficient. This moment translates into an apparent stylus force of $W_{x}=M_{W} / l$ and consequently into a virtual stylus bending of $x_{v}=W_{x} / c$ with $c$ being the stiffness of the stylus. For a two-sided probing, the friction force at the contacting points results thus in a virtual play of

$$
s_{v}=2 x_{v}=\frac{2\left|W_{x}\right|}{c}=\frac{2 r \mu F}{l c},
$$

which amounts for an assumed friction coefficient of $0.05<\mu<0.1$ and the numerical example given above to a virtual play in the range of $30 \mathrm{~nm}<s_{v}<60 \mathrm{~nm}$.

The friction only leads to hysteresis if the probe slides along the object surface. If the friction force can be absorbed by elastic deformation and the contact point sticks without slip on the surface, the virtual play will not harm and is taken into account if the measurement force is extrapolated to zero. A stick-slip effect and thus hysteresis between the stylus deflection and the measured displacement can be avoided by introducing some additional elasticity in the vertical direction and thus to let the sphere roll on the surface. This solution has been realized in the mechanical design of the probing system described

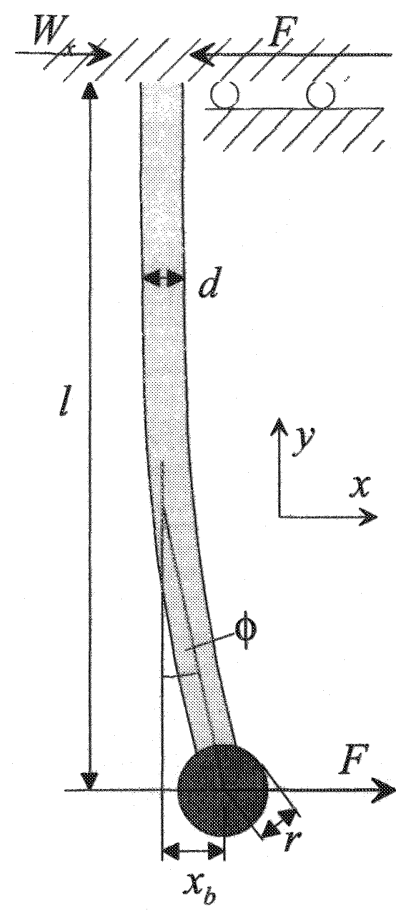

Figure 3: Stylus bending and applied forces. below.

\section{REALIZATION OF THE PROBE SYSTEM}

\subsection{Mechanical design}

The probe system has been realized by a monolithic flexure hinge design (Figure 4). It was optimized for stiffness and dynamic behavior. The mass of the moveable part was designed as small as possible in order to reduce the impact forces when touching the object with a reasonable impact velocity. Most of the mechanical machining was made by spark erosion. To achieve a good thermal stability, the structure was made out of invar, a low expansion alloy.

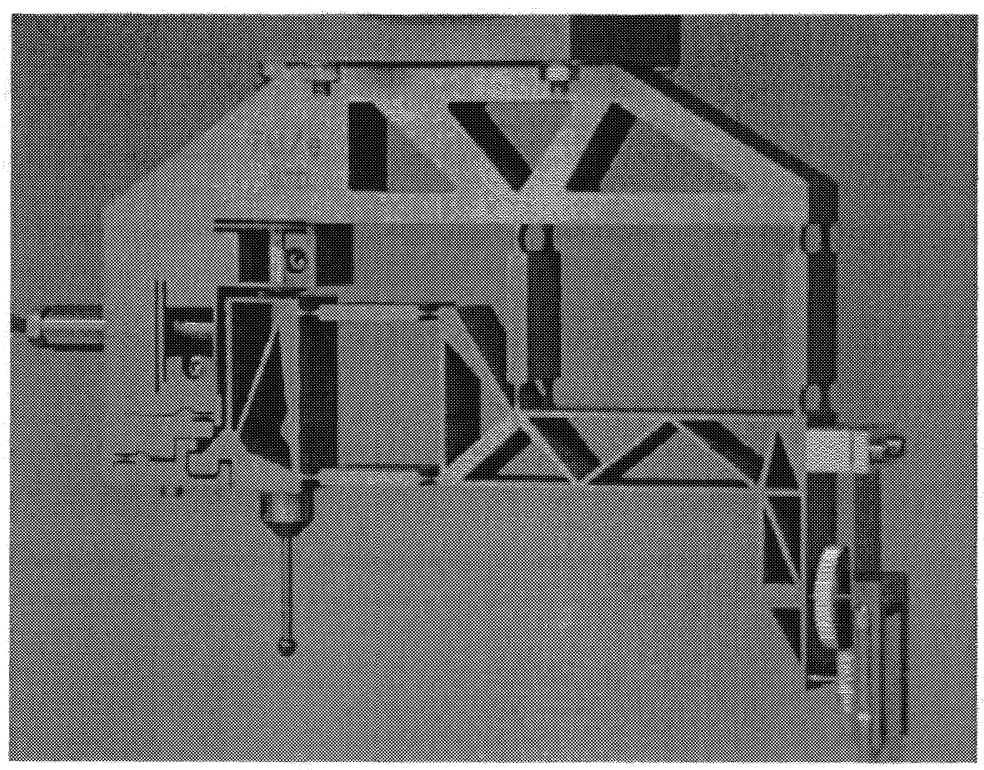

Figure 4: Flexure hinge probing system from invar, fabricated by electro erosion. 


\subsection{Measurement system and control}

As mentioned earlier, the measurement system for the horizontal displacement of the probe in the principal measurement direction is a plane mirror interferometer [4]. Its digital resolution is basically $5 \mathrm{~nm}$, but is in our case somewhat lower due to noise and averaging over several measurements.

The deflection of the hinge is measured with a capacitive probe [5], which provides $\pm 150 \mu \mathrm{m}$ range and a sensitivity of the analog output signal of $0.08 \mathrm{~V} / \mu \mathrm{m}$. Together with the digital voltmeter used for reading the output voltage of the sensor, a noise level of about $1 \mathrm{~nm}$ is obtained.

Figure 5 shows in detail the two capacitive probes for the deflection measurement in $\mathrm{x}$-and $\mathrm{z}$-direction. It shows also in the lower left corner an adjustable flexure hinge mechanism which provides adjustable limits for the deflection in both directions and a blocking mechanism for transportation.

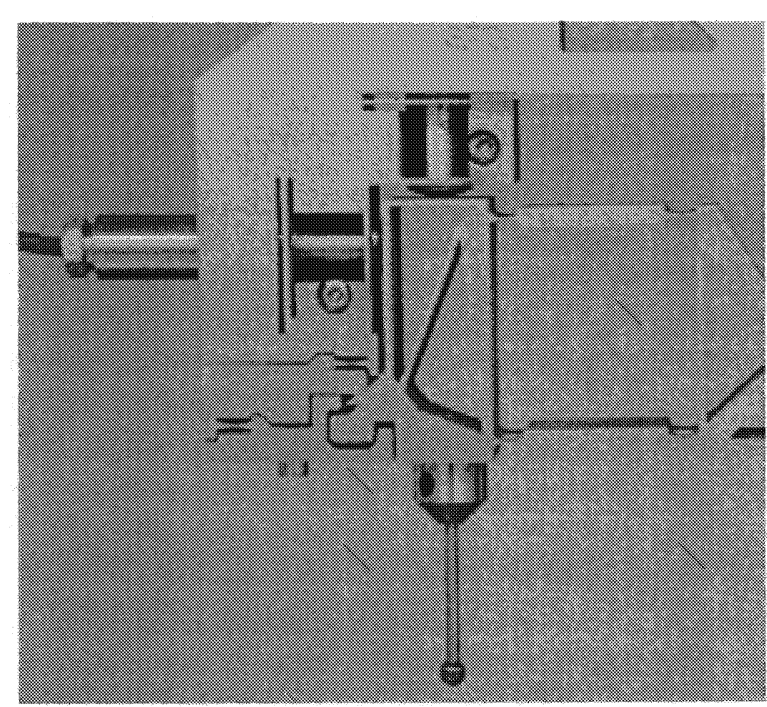

Figure 5: Capacitive sensors for the measurement of the hinge deflection and the measurement force.

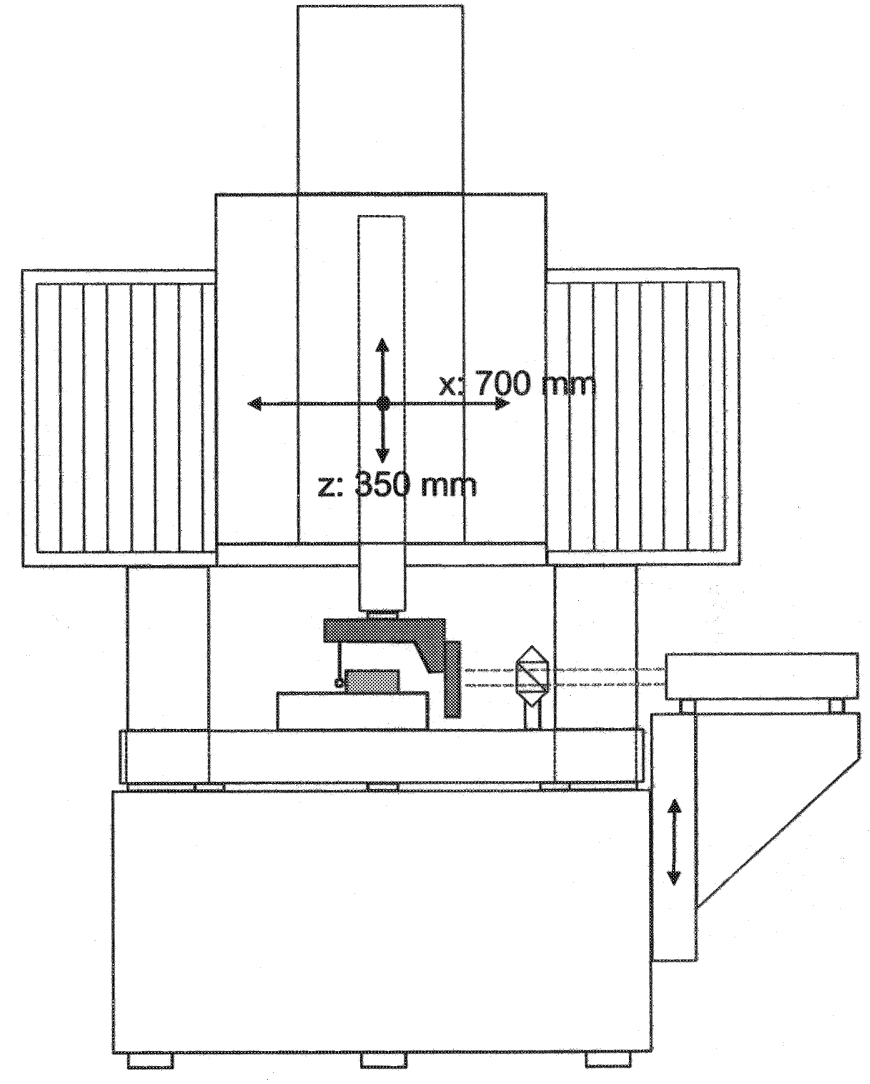

Figure 6: Measuring machine for the calibration of 1dimensional length standards.

The horizontal and vertical displacement axes of the measuring machine are both driven by brushless DC servo motors, controlled by incremental encoders on the motor axes. For the vertical axis, no further measurement system is used to measure the position, the incremental encoder and the backlash free drive result in a local positioning accuracy of about $1 \mu \mathrm{m}$, which is sufficient for repositioning the probe at the same measurement height after having moved around an external diameter standard.

The length measuring machine for which the probe was built is shown in Figure 6 and has been described elsewhere [1]. The upper half of the machine with the traverse and the ram is part of a co-ordinate measuring machine. All the rest was specially designed for 1-dimensional length measurement. It has a horizontal range of $700 \mathrm{~mm}$. With the probing system described here, the range of artefact size to be calibrated is limited by the spacing between the stylus and the mirror to about $100 \mathrm{~mm}$. The entire measurement process is numerically controlled by a PC with a software written in Visual Basic. 


\section{CHARACTERISATION OF THE PROBE SYSTEM}

\subsection{Sensitivity and linearity of the capacitive sensor}

The sensitivity and the linearity of the capacitive sensor has been calibrated by comparison with the laser interferometer. For this, the stylus was pushed in small steps to both, left and right direction, keeping the ram at a fixed position. Figure 6 shows the residuals from a linear fit for deflection to left and right direction, both measured inwards and outwards. The residuals are in a range of only $\pm 3 \mathrm{~nm}$ and mainly limited by the thermal stability, vibration and electronic noise.

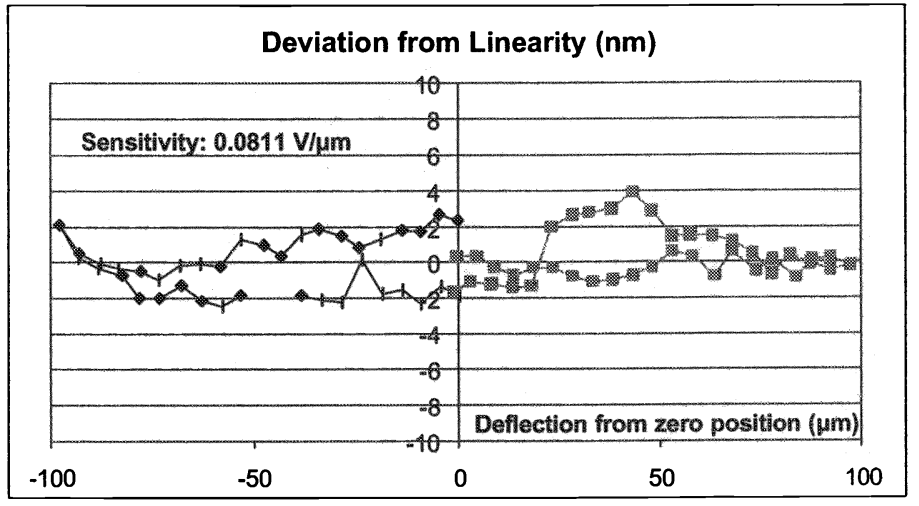

Figure 6: Deviation from linearity of the capacitive probe for a range of $\pm 100 \mu \mathrm{m}$.

\subsection{Measurement force and stiffness}

The stiffness of the flexure hinges was measured by contacting a force transducer with the probe sphere and measuring the deflection with the capacitive transducers, both in horizontal and vertical direction. The measurement results are shown in Figures 7. The stiffness of the hinges was determined to be $5.5 \cdot 10^{3} \mathrm{~N} / \mathrm{m}$ and $4.04 \cdot 10^{3} \mathrm{~N} / \mathrm{m}$ for the horizontal and vertical direction, respectively.
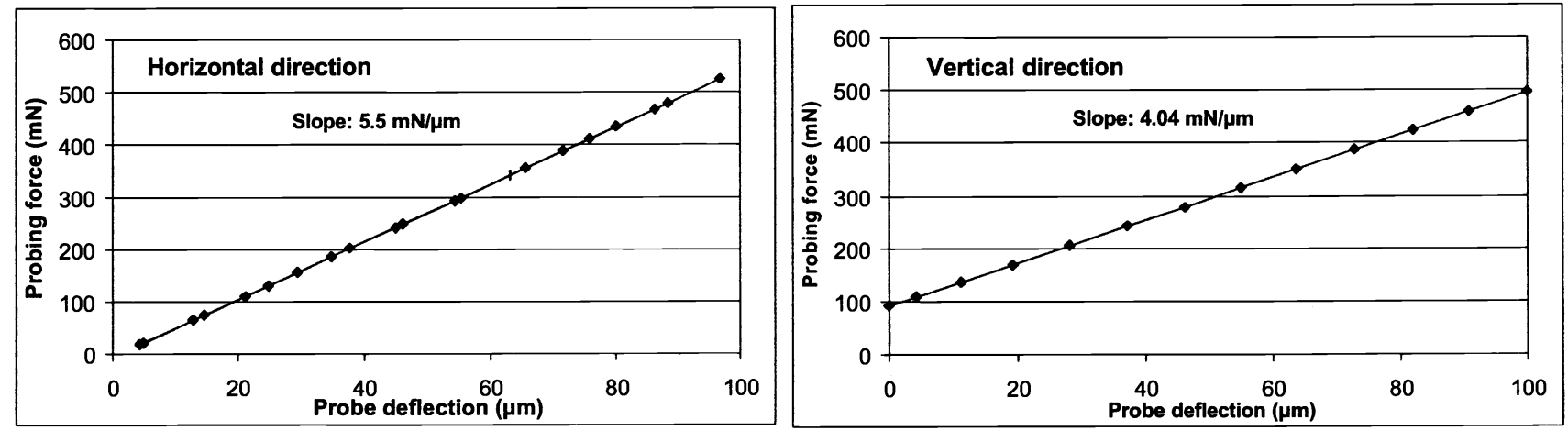

Figure 7: Measurement force and stiffness for horizontal and vertical deflection of the hinges.

The ratio of the stiffness between the stylus and the hinges can be determined by observing the ratio between the interferometer reading and the capacitive probe reading while moving the ram and contacting an object. For an infinitely stiff stylus, the interferometer reading would remain constant as soon as the object is contacted. Experimentally, a ratio of $1 / 18.9$ has been measured for the stylus used $(l=20 \mathrm{~mm}, d=2 \mathrm{~mm})$, i.e. for $100 \mu \mathrm{m}$ probe hinge deflection the stylus bent by $5.3 \mu \mathrm{m}$ (see Figure 9). It is by this ratio that the sensitivity requirements for the transducer system are reduced compared with the conventional system (see Sect. 2).

\subsection{Vertical deflection during contact}

As discussed in Section 3, the probing element is supposed to move in the vertical direction while contacting horizontally, due to roll of the probe on the surface, the circular movement of the hinges and any further crosstalk from the horizontal to the vertical movement. This effect has been measured with a second capacitive sensor. The Figure 8 shows that this movement is less than $1 \mu \mathrm{m}$ for $100 \mu \mathrm{m}$ horizontal deflection. Its asymmetric behaviour for probing to the left and right direction is not explained and probably due to asymmetry in the mechanical design. 

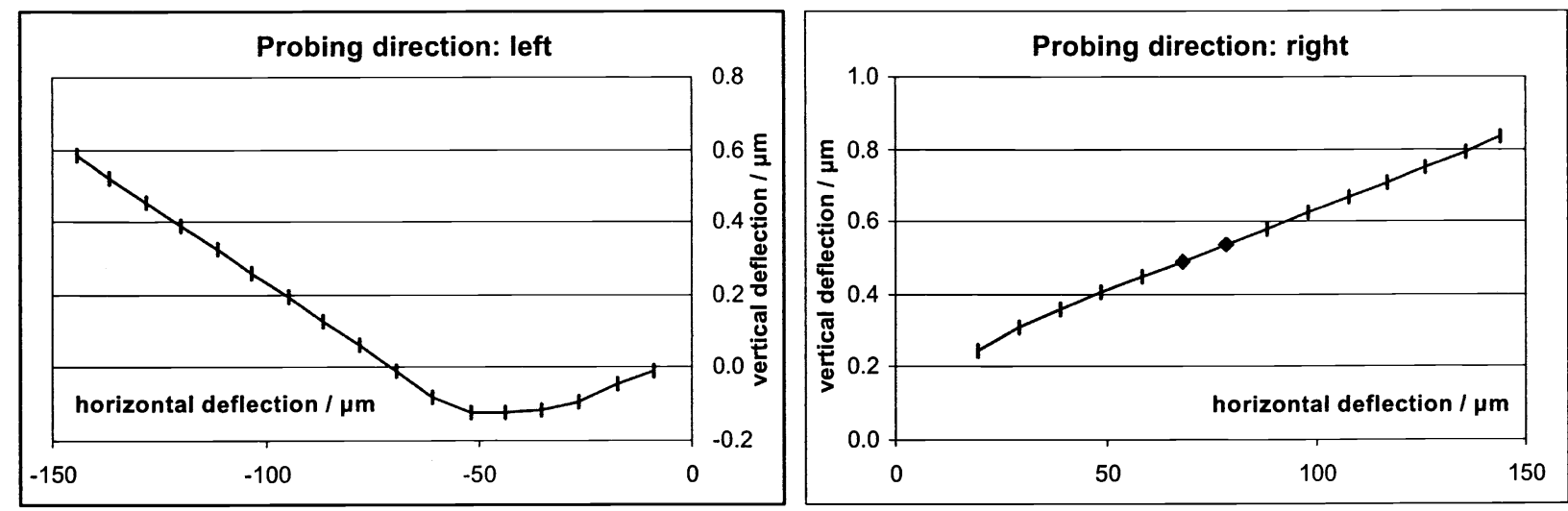

Figure 8: Vertical deflection of the hinges while probing horizontally to left and right direction.

Since the horizontal and the vertical stiffness of the hinges is approximately equal (see Sect. 5.2), the ratio between the forces corresponds to that of the deflection, i.e. about 1/150. Assuming the friction coefficient of the probe sphere on the contacted surface to be larger than 0.05 , the tangential force to be overcome by friction is in any case considerably larger than the elastic force of the hinge in the vertical direction, which ensures that not slip of the probing element on the surface will occur and thus any hysteresis due to stick-slip can be avoided.

\subsection{Extrapolation of measurement force to zero}

The ideal contact point to be determined is where the measurement force is extrapolated to the zero value. This is accomplished by contacting the surface at different probe deflections and extrapolating the function of the capacitive sensor versus interferometer reading to zero (zero crossing of the linear function with the y-axis in Figure 9). The determination of the zero point is expected to be accurate due to the small slope of the curve. Figure 10 shows the residuals from a linear fit of the probing curves obtained from 20 different contacting processes. Each probing process was realized by 9 deflection measurements in steps of about $9 \mu \mathrm{m}$ and a maximum deflection of $100 \mu \mathrm{m}$. In order to avoid vibration effects of running servo motors and timing problems, the single measurements were made quasistatically, i.e. the machine was stopped at each intermediate position. The average function (thick line) shows a slight non-linearity of about $10 \mathrm{~nm}$, with a standard deviation from the average function of less than $5 \mathrm{~nm}$.

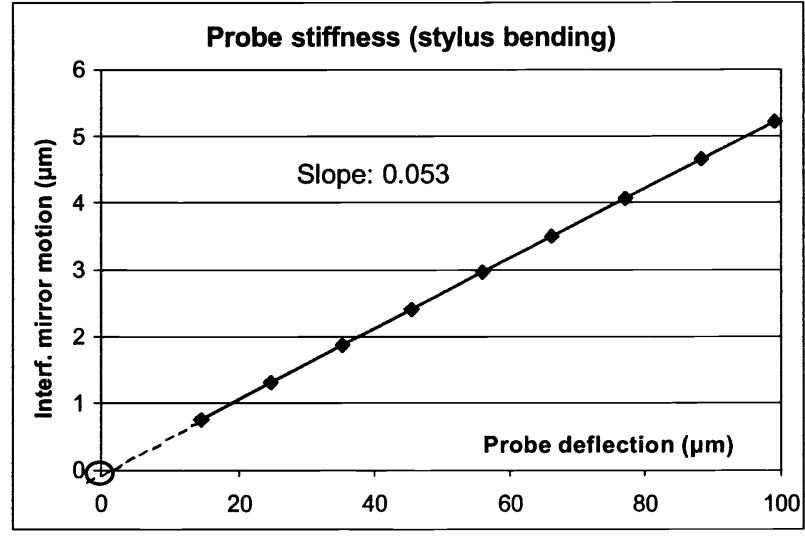

Figure 9: Probing function (interferometer vs. probe deflection reading) to be extrapolated to zero.

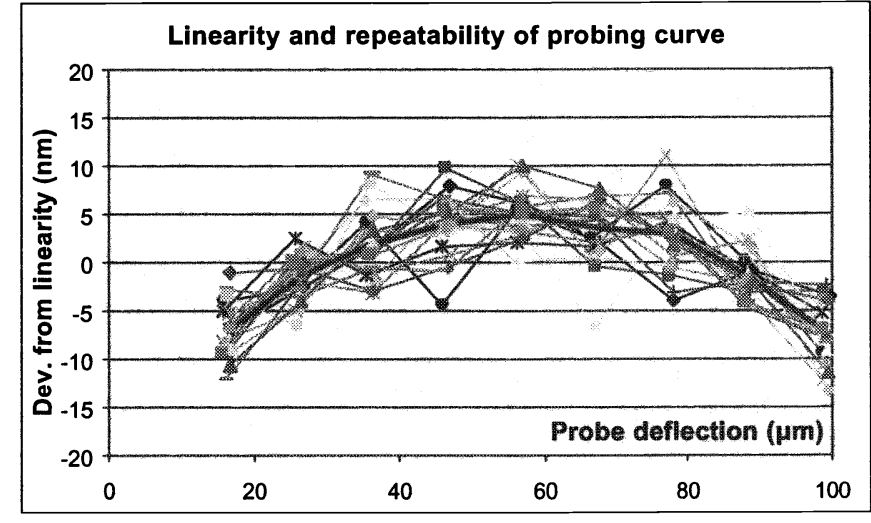

Figure 10: Residuals from linear fit of the probing function for 20 successive probings.

The repeatability of the extrapolated zero force contact point was determined from the standard deviation of 20 measurements and resulted in $5.4 \mathrm{~nm}$. It has to be noted, however, that a one-sided contact is a virtual point somewhere in the measurement volume of the machine and thus subject to mechanical instabilities, in particular thermal drift. Since 
using the multiple probing technique the measurement time for 20 repetitive probings was rather long (a few minutes), a linear drift was subtracted before calculating the above mentioned standard deviation.

\subsection{Probe constant and length measurements}

The probing system has been tested for the measurement of external size. For this a $5 \mathrm{~mm}$ gauge block was repeatedly measured. One measurement comprises a probing from the left and the right side and the measurement of the displacement of the probe head between the two surfaces of the gauge. Several series of 20 measurements were carried out. The standard deviation varied between $2.7 \mathrm{~nm}$ and $5.2 \mathrm{~nm}$ with an average of $3.7 \mathrm{~nm}$.

The absolute value and its accuracy obviously depends on the probe constant, which corresponds in fact to the diameter of the probing element. This is determined on a gauge block as well. The essential characteristics for an accurate probing system is therefore how well the probe constant determined on one length artefact can be transferred to the measurement of another artefact. For this, not only the repeatability but also the medium term stability of the probe constant is important. The latter showed a variation over several days with a standard deviation of $4 \mathrm{~nm}$. Part of this already small variation may be explained by long term temperature variations, since the thermal expansion of the probe sphere has not been taken into account. A $4 \mathrm{~nm}$ diameter variation of the ruby sphere would correspond to a temperature variation of about $0.2^{\circ} \mathrm{C}$.

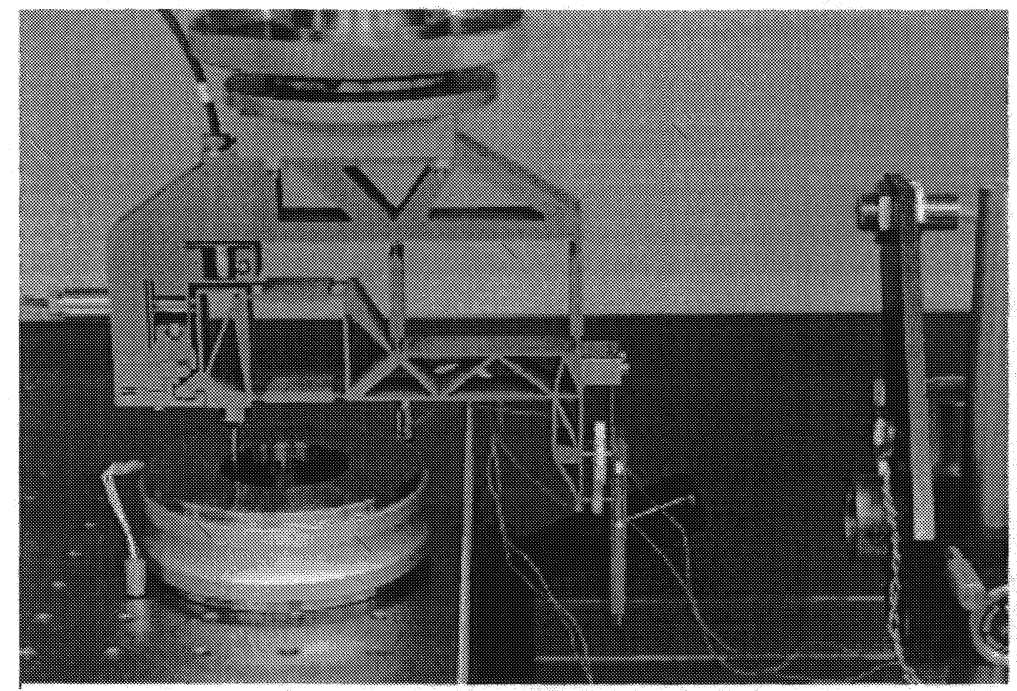

Figure 11: Novel probing system applied to internal diameter calibration of a ring gauge. At the right side, the interferometer optics is visible.

\section{CONCLUSIONS}

A novel probing system for 1-dimensional length measurements has been presented. The monolithic flexure hinge design provides a vertical degree of freedom, which avoids stick-slip effects along the measurement surface and thus any possible hysteresis. The large ratio of the stiffness between the stylus and the flexure hinges drastically reduces the requirements to the deflection/force transducer and allows thus for highly repeatable and accurate probing. The observed repeatability of the force free contact point and the medium term stability of the probe constant are both within a few nanometers and have demonstrated the suitability of the system for highly accurate length measurements.

\section{REFERENCES}

1. R. Thalmann, "A new high precision length measuring machine", 8e Congrès International de Métrologie, Besançon 1997.

2. F. Lüdicke, H.-J. Rademacher, "Bestimmung von Mass und Form an Zylindern und Kugeln", PTB-Mitteilungen 99, p. 429 (1989).

3. Diameter-measuring machine, Technical note, National Physical Laboratory, Teddington, UK (1984).

4. Hewlett Packard HeNe-Laser HP 5518A, High stability plane mirror interferometer HP 10706B.

5. Lion precision, Capacitive probe C1-C, Probe driver DMT 22. 